\title{
Noncommutative Field Theory and Lorentz Violation
}

\author{
Sean M. Carroll, ${ }^{1}$ Jeffrey A. Harvey, ${ }^{1}$ V. Alan Kostelecký, ${ }^{2}$ Charles D. Lane, ${ }^{3}$ and Takemi Okamoto ${ }^{1}$ \\ ${ }^{1}$ Enrico Fermi Institute, University of Chicago, Chicago, Illinois 60637 \\ ${ }^{2}$ Physics Department, Indiana University, Bloomington, Indiana 47405 \\ ${ }^{3}$ Physics Department, Colby College, Waterville, Maine 04901
}

(Received 9 May 2001; published 17 September 2001)

\begin{abstract}
The role of Lorentz symmetry in noncommutative field theory is considered. Any realistic noncommutative theory is found to be physically equivalent to a subset of a general Lorentz-violating standard-model extension involving ordinary fields. Some theoretical consequences are discussed. Existing experiments bound the scale of the noncommutativity parameter to $(10 \mathrm{TeV})^{-2}$.
\end{abstract}

DOI: 10.1103/PhysRevLett.87.141601

PACS numbers: 11.30.Cp, 02.40.Gh, 12.20.Fv

The idea that spacetime may intrinsically involve noncommutative coordinates has undergone a recent revival following the realization that this occurs naturally in string theory [1]. In this framework, the commutator of the coordinates $x^{\mu}$ in the spacetime manifold is

$$
\left[x^{\mu}, x^{\nu}\right]=i \theta^{\mu \nu},
$$

where $\theta^{\mu \nu}$ is real and antisymmetric. It is of interest to speculate that the physical world might involve noncommutative coordinates and to ask about current experimental sensitivity to putative realistic noncommutative quantum field theories.

The primary goal of this work is to study a physical issue that is central to any realistic noncommutative theory: the role of Lorentz symmetry. Violations of Lorentz symmetry are intrinsic to noncommutative theories by virtue of nonzero $\theta^{\mu \nu}$ in Eq. (1). Our study of these violations is motivated partly by theoretical progress in understanding the physics associated with Lorentz violation in ordinary quantum field theory and partly by recent experimental advances that make Lorentz tests among the most sensitive null experiments in existence [2].

One approach to constructing a noncommutative quantum field theory is to promote an established ordinary theory to a noncommutative one by replacing ordinary fields with noncommutative fields and ordinary products with Moyal $\star$ products, defined by

$$
\left.f \star g(x) \equiv \exp \left(\frac{1}{2} i \theta^{\mu \nu} \partial_{x^{\mu}} \partial_{y^{\nu}}\right) f(x) g(y)\right|_{x=y} .
$$

For gauge theories such as quantum electrodynamics (QED), ordinary gauge transformations must be modified to noncommutative generalizations. For noncommutative QED [3], the Hermitian Lagrangian is

$$
\mathcal{L}=\frac{1}{2} i \overline{\hat{\psi}} \star \gamma^{\mu} \overleftrightarrow{\hat{D}}_{\mu} \hat{\psi}-m \overline{\hat{\psi}} \star \hat{\psi}-\frac{1}{4 q^{2}} \hat{F}_{\mu \nu} \star \hat{F}^{\mu \nu}
$$

Here, carets indicate noncommutative quantities, $\hat{F}_{\mu \nu}=$ $\partial_{\mu} \hat{A}_{\nu}-\partial_{\nu} \hat{A}_{\mu}-i\left[\hat{A}_{\mu}, \hat{A}_{\nu}\right]_{\star}$, and $\hat{D}_{\mu} \hat{\psi}=\partial_{\mu} \hat{\psi}-i \hat{A}_{\mu} \star \hat{\psi}$, with $\hat{f} \star \overleftrightarrow{D}_{\mu} \hat{g} \equiv \hat{f} \star \hat{D}_{\mu} \hat{g}-\hat{D}_{\mu} \hat{f} \star \hat{g}$. Note that the inclusion of particles of charge other than 0 or \pm 1 is problematic [3]. This poses difficulties for a noncommutative generalization of the standard model, which would require other values for hypercharge assignments. In fact, noncommutative QED is similar to $\mathrm{U}(N)$ gauge theory as $N \rightarrow \infty$, and the allowed representations are the adjoint, fundamental, and antifundamental. In D-brane physics, adding two D-branes of charge 1 under a noncommutative $\mathrm{U}(1)$ leads to noncommutative $\mathrm{U}(2)$ gauge theory, which has non-Abelian $\mathrm{U}(2)$ gauge theory as its commutative limit instead of $U(1)$ with charge 2 .

The implementation of Lorentz transformations in a noncommutative theory is more involved than usual because the parameter $\theta^{\mu \nu}$ carries Lorentz indices. Two distinct types of Lorentz transformation exist [4]. For example, Eq. (3) is fully covariant under observer Lorentz transformations: rotations or boosts of the observer inertial frame leave the physics unchanged because both the field operators and $\theta^{\mu \nu}$ transform covariantly. However, these coordinate changes differ profoundly from rotations or boosts of a particle or localized field configuration within a fixed observer frame. The latter, called particle Lorentz transformations, leave $\theta^{\mu \nu}$ unaffected and hence modify the physics. This situation is closely analogous to the result of spontaneous Lorentz violation [5], with $\theta^{\mu \nu}$ playing the role of a tensor expectation value. In effect, $\theta^{\mu \nu}$ provides a four-dimensional directionality to spacetime in any fixed inertial frame. Any noncommutative theory therefore violates particle Lorentz symmetry.

The procedure leading to Eq. (3) lacks direct information about the identification of realistic physical variables with specific operators. For instance, the electron field $\hat{\psi}$ in the noncommutative QED (3) is itself noncommutative and obeys an unconventional gauge transformation law, so the identification of its quantum with the physical electron is nontrivial. Although it is presumably feasible in principle to calculate physical observables via noncommutative fields, we use here instead a correspondence between a noncommutative gauge theory and a conventional gauge theory, called the Seiberg-Witten map [6]. This permits the construction of an ordinary theory with ordinary 
gauge transformations having physical content guaranteed equivalent to the noncommutative theory.

The existence of an equivalent ordinary gauge theory for any realistic noncommutative theory involving noncommutative standard-model fields is of interest because there already exists a general extension of the ordinary standard model allowing for Lorentz violation $[4,7]$. This theory can be defined as the standard-model Lagrangian plus all possible gauge-invariant terms involving standard-model fields that preserve observer Lorentz invariance while breaking particle Lorentz symmetry. It therefore follows that any realistic noncommutative theory must be physically equivalent to a subset of the standard-model extension.

A variety of theoretical and experimental implications of the standard-model extension are known, and the existence of the equivalence ensures some of these also hold for any realistic noncommutative theory. The Lorentzviolating terms in the standard-model extension are contractions of field operators that transform as Lorentz tensors with coefficients that carry observer Lorentz indices. In any subset of this theory equivalent to a noncommutative theory, the coefficients for Lorentz violation must be expressed solely in terms of $\theta^{\mu \nu}$. This has several immediate consequences for any realistic noncommutative theory. As a simple example, energy and momentum are conserved in the full standard-model extension provided the coefficients for Lorentz violation are constant. Since $\theta^{\mu \nu}$ is independent of position, energy and momentum are conserved in any realistic noncommutative theory.

As another example, terms in the standard-model extension violate $C P T$ if and only if the coefficients for Lorentz violation carry an odd number of indices. Since it is impossible to construct such a coefficient from combinations of $\theta^{\mu \nu}$, it immediately follows that any realistic noncommutative theory necessarily preserves $C P T$. This generalizes a result obtained for the case of noncommutative QED [8]. In contrast, all other combinations of the discrete symmetries $C, P, T$ can be broken in a general noncommutative theory.

Further insight is provided by the observation that in a noncommutative field theory each factor of $\theta^{\mu \nu}$ is accompanied by two derivatives. Since bilinear fermion opera- tors in a noncommutative theory have mass dimension 3 or 4, the minimal dimension of the corresponding Lorentzviolating bilinear operators in the equivalent Lagrangian is 5 or 6 . In fact, higher-dimensional terms and nonlocal interactions are required for consistency at high scales in the full standard-model extension [9]. However, the absence here of Lorentz-violating operators of dimension 3 or 4 implies the fermionic sector of any realistic noncommutative theory is free of perturbative difficulties with stability and causality. This implies, for example, the absence of superluminal information transfer and conventional spin statistics.

Some noncommutative theories with $\theta^{0 j} \neq 0$ exhibit difficulties with perturbative unitarity [10], but ones with only $\theta^{j k}$ nonzero are acceptable. Since a theory with $\theta^{0 j} \neq 0, \theta_{\mu \nu} \theta^{\mu \nu}>0$, and $\theta_{\mu \nu} \tilde{\theta}^{\mu \nu}=0$ can be converted into one with only $\theta^{j k}$ nonzero by a suitable observer Lorentz transformation, the presence of observer Lorentz invariance implies that there are no difficulties with perturbative unitarity provided $\theta_{\mu \nu} \theta^{\mu \nu}>0, \theta_{\mu \nu} \tilde{\theta}^{\mu \nu}=0$, which allows certain cases with $\theta^{0 j} \neq 0$. A similar condition presumably applies for open bosonic strings, where the presence of a nonzero $B^{j k}$ field is known to be equivalent to a constant magnetic field on a $\mathrm{D} p$ brane [6]. In the standard-model extension, Lorentz-violating operators with extra time-derivative couplings do cause some interpretational difficulties, but these can be handled by redefining the fields to evolve canonically $[9,11]$. We expect analogous methods to apply for noncommutative theories with $\theta^{0 j} \neq 0$.

For definiteness, we focus primarily on the noncommutative QED (3) with $\theta_{\mu \nu} \theta^{\mu \nu}>0$ in the remainder of this Letter. In this case, the explicit form of the Seiberg-Witten map is known to lowest order in $\theta^{\mu \nu}[6,12]$ :

$$
\begin{aligned}
\hat{A}_{\mu} & =A_{\mu}-\frac{1}{2} \theta^{\alpha \beta} A_{\alpha}\left(\partial_{\beta} A_{\mu}+F_{\beta \mu}\right), \\
\hat{\psi} & =\psi-\frac{1}{2} \theta^{\alpha \beta} A_{\alpha} \partial_{\beta} \psi .
\end{aligned}
$$

This leading-order form suffices for many purposes, since any physical noncommutativity in nature must be small.

Substitution of the solution (4) into Eq. (3) and applying the definition (2) yields the ordinary quantum field theory that is physically equivalent to noncommutative QED to leading order in $\theta^{\mu \nu}$ :

$$
\begin{aligned}
\mathcal{L}= & \frac{1}{2} i \bar{\psi} \gamma^{\mu} \overleftrightarrow{D}_{\mu} \psi-m \bar{\psi} \psi-\frac{1}{4} F_{\mu \nu} F^{\mu \nu}-\frac{1}{8} i q \theta^{\alpha \beta} F_{\alpha \beta} \bar{\psi} \gamma^{\mu} \overleftrightarrow{D}_{\mu} \psi+\frac{1}{4} i q \theta^{\alpha \beta} F_{\alpha \mu} \bar{\psi} \gamma^{\mu} \overleftrightarrow{D}_{\beta} \psi+\frac{1}{4} m q \theta^{\alpha \beta} F_{\alpha \beta} \bar{\psi} \psi \\
& -\frac{1}{2} q \theta^{\alpha \beta} F_{\alpha \mu} F_{\beta \nu} F^{\mu \nu}+\frac{1}{8} q \theta^{\alpha \beta} F_{\alpha \beta} F_{\mu \nu} F^{\mu \nu} .
\end{aligned}
$$

In this equation, we have redefined the gauge field $A_{\mu} \rightarrow$ $q A_{\mu}$ to display the charge coupling of the physical fermion, and $D_{\mu} \psi=\partial_{\mu} \psi-i q A_{\mu} \psi$ as usual.

The expression (5) is manifestly gauge invariant. It consists of ordinary QED plus nonrenormalizable Lorentzviolating corrections and is therefore a subset of the QED limit of the standard-model extension, as expected. However, many terms allowed in the latter theory are absent, in- cluding all those that violate $C P T$. Note that the $\gamma$-matrix structure in Eq. (5) is inherited from the usual one in Eq. (3), so no couplings to axial-vector or tensor bilinears appear. Note also that all noncommutative effects vanish for neutral fermions.

With this explicit theory in hand, we can consider some possible experimental implications of noncommutativity. 
Here, we focus attention on the case of experiments involving constant electromagnetic fields. For this purpose, it is useful to extract from the theory (5) an effective Lagrangian describing the leading-order effects of noncommutativity in constant electromagnetic fields. We therefore make the replacement $F_{\mu \nu} \rightarrow f_{\mu \nu}+F_{\mu \nu}$, where $f_{\mu \nu}$ is understood to be a constant background field and $F_{\mu \nu}$ now denotes a small dynamical fluctuation.

Keeping only terms up to quadratic order in the fluctuations and performing a physically irrelevant rescaling of the fields $\psi$ and $A_{\mu}$ to maintain conventionally normalized kinetic terms yields the Hermitian Lagrangian

$$
\begin{aligned}
\mathcal{L}= & \frac{1}{2} i \bar{\psi} \gamma^{\mu} \overleftrightarrow{D}_{\mu} \psi-m \bar{\psi} \psi-\frac{1}{4} F_{\mu \nu} F^{\mu \nu} \\
& +\frac{1}{2} i c_{\mu \nu} \bar{\psi} \gamma^{\mu} \overleftrightarrow{D}^{\nu} \psi-\frac{1}{4} k_{F \alpha \beta \gamma \delta} F^{\alpha \beta} F^{\gamma \delta}
\end{aligned}
$$

In this equation, the charge $q$ in the covariant derivative is replaced with a scaled effective value [13]

$$
q_{\mathrm{eff}}=\left(1+\frac{1}{4} q f^{\mu \nu} \theta_{\mu \nu}\right) q .
$$

The dimensionless coefficients $c_{\mu \nu}$ and $k_{F \alpha \beta \gamma \delta}$ are

$$
\begin{aligned}
c_{\mu \nu}= & -\frac{1}{2} q f_{\mu}{ }^{\lambda} \theta_{\lambda \nu}, \\
k_{F \alpha \beta \gamma \delta}= & -q f_{\alpha}{ }^{\lambda} \theta_{\lambda \gamma} \eta_{\beta \delta}+\frac{1}{2} q f_{\alpha \gamma} \theta_{\beta \delta} \\
& -\frac{1}{4} q f_{\alpha \beta} \theta_{\gamma \delta}-(\alpha \leftrightarrow \beta)-(\gamma \leftrightarrow \delta) \\
& +(\alpha \beta \leftrightarrow \gamma \delta) .
\end{aligned}
$$

The notation here is that of the standard-model extension in its QED limit [4]. Of the ten types of term allowed in the latter theory, six are excluded here by $C P T$ symmetry and two by the requirement of no couplings to axial or tensor fermion bilinears. Note that $c_{\mu \nu}, k_{F \alpha \beta \gamma \delta}$ depend linearly on the background electric and magnetic fields, as required for dimensional consistency.

In the photon sector, there are presently no published bounds on the coefficients $k_{F \alpha \beta \gamma \delta}$. The modified Maxwell equations in vacuo have been studied, and it appears feasible to place bounds at the scale of about $10^{-28}$ on certain components of $k_{F \alpha \beta \gamma \delta}$, using measurements of the birefringence of radiation from cosmological sources [4,14]. However, the dependence of $k_{F \alpha \beta \gamma \delta}$ on the minuscule intergalactic magnetic field and the likely dilution of any effect due to random field orientations implies only weak bounds on $\theta^{\mu \nu}$ are likely.

Instead, we turn to the fermion sector. Numerous tests of Lorentz violation have been performed in the context of the standard-model extension, but many of them can detect only $C P T$ violation or anomalous spin couplings and so place no bounds on $c_{\mu \nu}$. One class of tests with sensitivity to $c_{\mu \nu}$ involves the recent clock-comparison experiments [15]. These monitor the difference between two atomic hyperfine or Zeeman transition frequencies, searching for variations as the Earth rotates. The existing analysis [16] of the implications of these experiments can be adapted to the present situation.

The energy shift $\delta$ in an atomic state labeled $\left|F, m_{F}\right\rangle$ can be calculated as the expectation value of the Hermitian perturbation Hamiltonian obtained from Eq. (6). It has the form $\delta \sim \tilde{m}_{F} \sum_{w} \gamma_{w} \tilde{c}_{q}^{w}$, where $\tilde{m}_{F}$ is a ratio of ClebschGordan coefficients, $w$ labels the particle species (electron, proton, neutron) of mass $m_{w}$ and charge $q_{w}, \gamma_{w}$ is an expectation value of momentum operators in an extremal state of the atomic or nuclear submanifold of levels, and $\tilde{c}_{q}^{w} \equiv m_{w}\left(c_{11}+c_{22}-2 c_{33}\right)$ is a quadrupole combination of coefficients for Lorentz violation. Expressions for $\tilde{m}_{F}$ and $\gamma_{w}$ are provided in Eqs. (7) and (10) of Ref. [16]. With the magnetic field $f^{12} \equiv-B$ along the 3 axis in the laboratory frame, we find $c_{q}^{w}=m_{w} q_{w} B \theta^{12}$.

In the laboratory frame rotating with the Earth, the parameters $m_{w}, q_{w}$, and $B$ are fixed but $\theta^{12}$ varies with time $t$. To display the corresponding $t$ dependence of the energy shift $\delta$, we use a nonrelativistic transformation to convert to nonrotating coordinates $(X, Y, Z)$ compatible with celestial equatorial coordinates [16]. This gives

$$
\delta=E_{0}+E_{1 X} \cos \Omega t+E_{1 Y} \sin \Omega t,
$$

where $\Omega$ is the Earth's sidereal rotation frequency, $E_{0}$ is an irrelevant constant, and

$$
\left(E_{1 X}, E_{1 Y}\right)=\tilde{m}_{F} B \sin \chi \sum_{w} q_{w} m_{w} \gamma_{w}\left(\theta_{Y Z}, \theta_{Z X}\right),
$$

with $\chi$ the angle between the 3 and the $Z$ axes. Note that, despite its quadrupole nature, the energy shift $\delta$ is periodic in $\Omega t$. This contrasts with the $2 \Omega t$ dependence arising when $c_{\mu \nu}$ is independent of $B$.

We can apply these results to recent clock-comparison tests [15]. Most place bounds on variations with $\Omega t$ but in the context of the nuclear valence model are sensitive only to effects from the neutron, which vanish here because the neutron is uncharged. The exception is the experiment of Berglund et al., which compares transitions in ${ }^{199} \mathrm{Hg}$ and ${ }^{133}$ Cs. In this experiment, as in the others, the electronic angular momentum is $J \leq 1 / 2$, so $\gamma_{e}$ vanishes and there is no signal associated with the electron. However, the nuclear spin of the ${ }^{133} \mathrm{Cs}$ atom is $I=7 / 2$, and the nuclear valence model predicts the valence nucleon to be a proton, so the experimental limit of about $100 \mathrm{nHz}$ on possible sidereal variations yields a bound $\left|\theta^{Y Z}\right|,\left|\theta^{Z X}\right| \lesssim(10 \mathrm{GeV})^{-2}$.

The above bound is suppressed due to the weak magnetic field $(B \sim 5 \mathrm{mG})$ used in the experiment. In contrast, the experiment of Prestage et al. involves an applied field of about $1 \mathrm{~T}$. It is therefore worth considering possible effects outside the valence model. The explicit value of $\gamma_{p}$ in Eq. (10) is an expectation value of momentum operators in the multiparticle wave function $|\psi\rangle$ for the ${ }^{9} \mathrm{Be}$ nucleus used in the experiment [17]:

$$
|\psi\rangle=C_{1}\left({ }^{1} S,{ }^{2} P\right)+C_{2}\left({ }^{1} D,{ }^{2} P\right)+C_{3}\left({ }^{1} D,{ }^{2} D\right) .
$$


Each term in parentheses refers to the proton and neutron spin and orbital angular momentum according to $\left({ }^{2 S_{p}+1} L_{p},{ }^{2 S_{n}+1} L_{n}\right)$, and the coefficients are $C_{1} \simeq 0.731$, $C_{2} \simeq-0.344, C_{3} \simeq-0.589$. A calculation gives

$$
\gamma_{p}=-\left[7\left(C_{2}^{2}-C_{3}^{2}\right)+8 \sqrt{5} C_{1} C_{2}\right] K_{p} / 150 .
$$

Here, $\quad K_{p}=\left\langle p^{2}\right\rangle_{p} / m_{p}^{2} \simeq 10^{-2}$, yielding $\gamma_{p} \simeq 5 \times$ $10^{-4}$. Using the results of Prestage et al., we find

$$
\left|\theta^{Y Z}\right|,\left|\theta^{Z X}\right| \lesssim(10 \mathrm{TeV})^{-2},
$$

as a conservative limit [18].

Other low-energy bounds on $\theta^{\mu \nu}$ exist. Measurements of the Lamb shift give [19] a bound several orders of magnitude weaker than (13). A speculative bound some 20 orders of magnitude stronger than (13) has been claimed [20] from an analysis of clock-comparison experiments. This analysis finds terms with anomalous spin couplings and obtains a bound by supposing that, in an eventual formulation of noncommutative quantum chromodynamics, such couplings would produce a coherent effect involving the nuclear force.

For bounds on $c_{\mu \nu}$, high-energy experiments appear to provide no particular advantage over low-energy ones, basically because the effects scale with momentum similar to those from the usual fermion kinetic term [21]. Assuming the interactions in Eq. (5) affect at least some high-energy cross sections, the attainable high-energy bound can be crudely estimated as about $(1 \mathrm{TeV})^{-2}$ by noting that leading-order couplings involving $\theta^{\mu \nu}$ come with two powers of momentum, while cross sections at $100 \mathrm{GeV}$ are typically known to no better than about $1 \%$. This bound is compatible with existing analyses [22].

Further theoretical analysis might improve the bound (13). For example, it may be worth studying the effect of the magnetic field at the nucleus caused by atomic electrons, since under suitable circumstances the effect of this field in $c_{\mu \nu}$ might dominate the applied one. Also, additional experimental sensitivity might arise if the neutron is coupled in the adjoint representation of noncommutative QED. The range of relevant tests might be further broadened if additional $\gamma$-matrix structures arise in radiative corrections in the theory (5) or in more complicated versions of noncommutative QED obtained from the radiative effective action in ordinary QED.

Several experimental options exist for improving the bound (13). One would be to perform a clock-comparison test in a large field using substances that are particularly favorable for theoretical calculations. These include the subset of species listed in Table III of Ref. [16] that have quadrupole sensitivity to proton effects. It would be ideal to compare one such species to a reference for which noncommutative effects are absent. For example, an experiment comparing transitions in ${ }^{209} \mathrm{Bi}$ with ${ }^{3} \mathrm{He}$ or, perhaps more feasibly, ${ }^{87} \mathrm{Rb}$ with ${ }^{3} \mathrm{He}$ has the potential to provide an improved reliable bound on $\theta^{\mu \nu}$.
This work was supported in part by DOE Grant No. DEFG01-91ER40661, NSF Grant No. 9901194, the Packard Foundation, and the Sloan Foundation.

[1] A. Connes, M. Douglas, and A. Schwarz, J. High Energy Phys. 02, 003 (1998). For recent reviews, see N. A. Nekrasov, hep-th/0011095; A. Konechny and A. Schwarz, hep-th/0012145; J. A. Harvey, hep-th/0102076.

[2] Some reviews are in CPT and Lorentz Symmetry, edited by V. A. Kostelecký (World Scientific, Singapore, 1999).

[3] M. Hayakawa, Phys. Lett. B 478, 394 (2000).

[4] D. Colladay and V. A. Kostelecký, Phys. Rev. D 55, 6760 (1997); 58, 116002 (1998).

[5] V. A. Kostelecký and S. Samuel, Phys. Rev. D 39, 683 (1989); 40, 1886 (1989); 63, 224 (1989); V. A. Kostelecký and R. Potting, Nucl. Phys. B359, 545 (1991); Phys. Lett. B 381, 89 (1996).

[6] N. Seiberg and E. Witten, J. High Energy Phys. 09, 032 (1999).

[7] V. A. Kostelecký and R. Potting, Phys. Rev. D 51, 3923 (1995)

[8] M. M. Sheikh-Jabbari, Phys. Rev. Lett. 84, 5265 (2000).

[9] V. A. Kostelecký and R. Lehnert, Phys. Rev. D 63, 065008 (2001).

[10] J. Gomis and T. Mehen, Nucl. Phys. B591, 265 (2000).

[11] R. Bluhm, V. A. Kostelecký, and N. Russell, Phys. Rev. Lett. 79, 1432 (1997); Phys. Rev. D 57, 3932 (1998).

[12] A. A. Bichl et al., hep-th/0102103.

[13] The effective charge varies with the applied field. In a $1 \mathrm{~T}$ magnetic field with the maximum possible $\theta^{j k}, q_{\text {eff }}$ differs by only about a part in $10^{24}$ from its zero-field value, which is presently unobservable.

[14] This technique was used to constrain the photon-sector coefficient $k_{A F \mu}$ at the level of $10^{-42} \mathrm{GeV}$ : S. M. Carroll, G. B. Field, and R. Jackiw, Phys. Rev. D 41, 1231 (1990).

[15] J. D. Prestage et al., Phys. Rev. Lett. 54, 2387 (1985); S. K. Lamoreaux et al., Phys. Rev. Lett. 57, 3125 (1986); Phys. Rev. A 39, 1082 (1989); T.E. Chupp et al., Phys. Rev. Lett. 63, 1541 (1989); C. J. Berglund et al., Phys. Rev. Lett. 75, 1879 (1995); D. Bear et al., Phys. Rev. Lett. 85, 5038 (2000).

[16] V. A. Kostelecký and C. D. Lane, Phys. Rev. D 60, 116010 (1999); J. Math. Phys. 40, 6245 (1999).

[17] M. E. Rose and H. A. Bethe, Phys. Rev. 51, 205 (1937).

[18] Using an exact relativistic transformation between the laboratory and nonrotating frames in deriving Eq. (10) reveals contributions from components $\theta^{0 J}$ that are suppressed by factors of about $10^{-6}$. We therefore also obtain bounds of roughly $(10 \mathrm{GeV})^{-2}$ on these components, although an accurate treatment requires incorporation of subleading effects disregarded here.

[19] M. Chaichian, M. M. Sheikh-Jabbari, and A. Tureanu, Phys. Rev. Lett. 86, 2716 (2001).

[20] I. Mocioiu, M. Pospelov, and R. Roiban, Phys. Lett. B 489, 390 (2000).

[21] D. Colladay and V. A. Kostelecký, Phys. Lett. B 511, 209 (2001).

[22] J. Hewett, F. Petriello, and T. Rizzo, hep-ph/0010354; P. Mathews, Phys. Rev. D 63, 075007 (2001). 